\title{
Lowered ultraviolet minimal erythema dose in hemiplegia
}

\author{
Neil H. Cox* and Sharon J. Williams \\ Clatterbridge Hospital, Bebington, Wirral, Merseyside, L63 4JY, UK.
}

\begin{abstract}
Summary: In view of recent reports of increased tanning in hemiplegic limbs, we have investigated ultraviolet (u.v.) minimal erythema dose (MED) in hemiplegia using the bilateral comparison technique. Seven of 10 patients had a lower MED in the hemiplegic arm compared to the normal side, the mean reduction being $16 \%$ (range $0-33 \%, P=0.003$ ). No patients had a higher MED in the hemiplegic arm. We review the literature regarding other non-neurological features of hemiplegia, in particular asymmetry of temperature, oedema, and finger clubbing, and we propose a vasomotor or trophic aetiology for these findings.
\end{abstract}

\section{Introduction}

A number of clinical features of hemiplegia have been reported in addition to the overtly neurological alterations in tone, power, sensation and coordination. These include unilateral oedema, finger clubbing, and asymmetrical axillary temperature. Exton-Smith \& Crockett (1957) reported oedema, especially of the arm, in $16 \%$ of their patients with hemiplegia, whilst Mulley (1980) found that $47 \%$ of his series of 30 patients had an axillary temperature difference of $0.5^{\circ} \mathrm{C}$ or greater, the hemiplegic side being cooler in all the patients with this degree of asymmetry. Although finger clubbing is less easy to quantify, Alvarez et al. (1975) reported this in 12 of $87(14 \%)$ of patients with 'obvious' hemiplegia, and Denham et al. (1975) felt that clubbing can be seen in the majority of patients with long-standing hemiplegia.

Uneven tanning in hemiplegia has recently been reported ('Any Questions' British Medical Journal, 1983; Williams, 1984) following sunlight exposure, and cannot be explained by asymmetrical ultraviolet light (UVL) exposure as Cox (1984) has documented the same phenomenon in a hemiplegic patient having PUVA therapy. In all these cases the hemiplegic side showed increased tanning.

Because oedema, clubbing, and skin temperature may all have a vascular basis, and in view of the evidence for altered cutaneous response to UVL, we have investigated the erythema response to UVL which is due to vasodilatation.

\footnotetext{
*Correspondence and present address: N.H. Cox, B.Sc., M.R.C.P., Department of Dermatology, Western Infirmary, Glasgow, G11 6NT, UK.

Accepted: 7 February 1985
}

\section{Materials and methods}

Ten patients with hemiplegia of 2 weeks to $32 y$ duration were studied, none of whom had any obvious cutaneous asymmetry of the ventral forearm. All gave informed consent.

A Kromayer Model 10S lamp (Hanovia Ltd, Slough) was used as the UVL source. In view of the wide range of spectral emission lines produced by this apparatus, the minimal erythema dose (MED) was recorded in terms of duration of exposure rather than energy per unit area.

Ventral forearm was used for MED determination. Exposure to UVL, and later assessment of erythema, was simultaneous for each arm in any individual. This bilateral comparison technique thus uses the normal arm as a control for the hemiplegic arm, and eliminates error due to sequential testing or due to interindividual differences (such as skin colour).

There are many different definitions of MED used by different authors (Hawk \& Parrish, 1982), the most common being the least dose of u.v. radiation which produces erythema with sharp margins, corresponding to the area exposed. The time between exposure and assessment is usually 7-24 h (Hawk \& Parrish, 1982). Our criteria for minimal erythema dose were that areas of erythema should have a sharp margin when assessed at $7 \mathrm{~h}$. All sites of u.v. exposure had the same diameter $(1 \mathrm{~cm})$. As many patients had obvious hemiplegia, true 'blind' assessment was precluded, but the number of sites irradiated was the same on each arm of any individual. Bilateral axillary temperature was also recorded.

Statistical significance of differences between the two sides was assessed by the $t$ test for paired comparisons. 


\section{Results}

Seven of our 10 patients had a reduced MED in the hemiplegic arm compared to the unaffected side, the other three having symmetrical results (Figure 1). The mean reduction in MED was $16 \%$ (range $0-33 \%$ ) which was highly significant $(P=0.003)$.

Axillary temperature of the hemiplegic side was, in comparison to the normal side, reduced in 5 patients (by $0.1-0.5^{\circ} \mathrm{C}$ ), increased in 2 (by $0.1-0.2^{\circ} \mathrm{C}$ ), and symmetrical in 3 patients. These results were not statistically significant.

There was no statistically significant correlation between reduced MED and either temperature asymmetry or duration of hemiplegia, although both MED and temperature were symmetrical in the patient with hemiplegia of 2 weeks duration, and the greatest degree of symmetry in both parameters was in the patient who had been hemiplegic for $32 \mathrm{y}$.

\section{Discussion}

It seems likely that vasomotor or trophic neurological changes are the cause of many of the features seen in hemiplegic limbs. Altered temperature is most easily explained by vascular changes, and it is interesting that of the 12 patients with unilateral finger clubbing and hemiplegia described by Alvarez et al. (1975), the skin of the affected side was cooler in at least 9 cases. Vasomotor and trophic changes are typical of the shoulder-hand syndrome which may occasionally occur as a consequence of hemiplegia (Steinbrocker \& Argyros, 1958). Skin atrophy, oedema, and other cutaneous changes such as hyperhidrosis and hypertrichosis, may all occur in this syndrome.

The apparent contradiction of lowered MED (implying an increase in vasodilatation in response to u.v. irradiation) and the frequently demonstrated lowered axillary temperature (suggesting decreased skin blood flow) could be explained if the problem is one of impaired vasomotor control. An alternative explanation is suggested by the observation of Williams (1984) who believes, on the basis of personal experience, that UVL induced skin thickening may be impaired in hemiplegia. Epidermal hyperplasia, particularly thickening of the stratum corneum, is a well documented response to UVL, and leads to increased tolerance to UVL (Hawk \& Parrish, 1982). If skin thickening due to UVL is impaired in hemiplegia, then increased erythema and tanning would occur in response to

\section{References}

ALVAREZ, A.S., McNAIR, D., WILDMAN, J. \& HEWSON, J.W. (1975). Unilateral clubbing of the fingernails in patients with hemiplegia. Gerontologia Clinica (Basel), 17, 1.

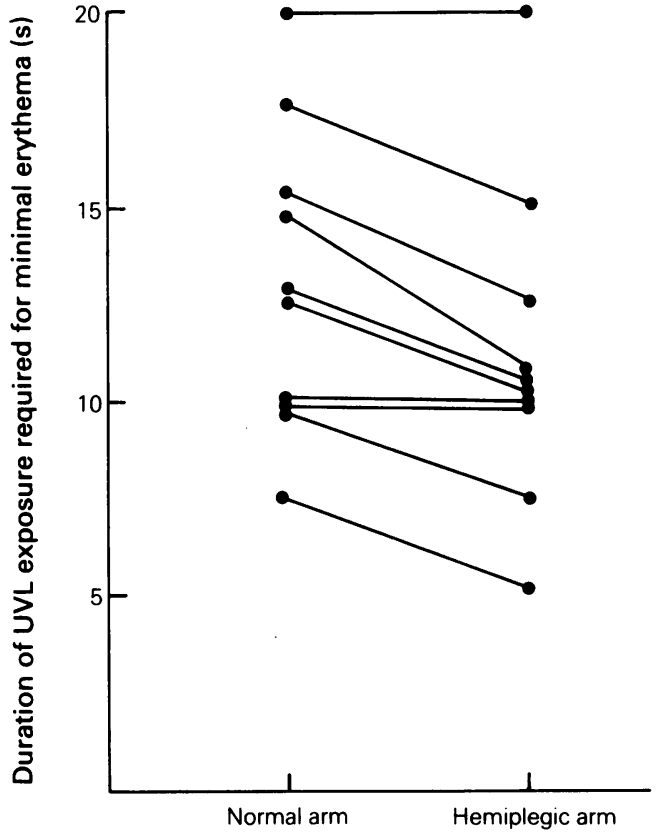

Figure 1 Bilateral comparison of minimal erythema dose of UVL in normal and hemiplegic limbs $(P=0.003)$

UVL. Altered vasomotor control and impaired skin thickening in response to UVL are not mutually exclusive, and could both be involved in causing a lowered MED in hemiplegia.

We have therefore presented evidence for asymmetry of u.v. erythema in hemiplegia, and suggest that asymmetry of oedema, finger clubbing, skin temperature, and MED may all be due to vasomotor changes in hemiplegic limbs. However, it is clear that the exact alteration in cutaneous responses to UVL following hemiplegia is poorly understood and warrants further investigation. Two potential implications for hemiplegic patients are worthy of discussion, although as far as we are aware neither has been reported. Firstly, as sunburn is severe solar u.v. erythema, our findings suggest that sunburn might be increased in hemiplegic limbs. It is possible that such cases have occurred but have been blamed on altered sensation or poor mobility of the hemiplegic limb. Secondly, as a tendency to sunburn is a risk factor for melanoma, there is a theoretical risk that this might be increased in hemiplegia.

ANY QUESTIONS? (1983). British Medical Journal, 287, 1708. COX, N.H. (1984). Asymmetrical tanning in hemiplegia. The Practitioner, 228, 1106. 
DENHAM, M.J., HODKINSON, H.M. \& WRIGHT, B.M. (1975). Unilateral clubbing in hemiplegia. Gerontologia Clinica (Basel), 17, 7.

EXTON-SMITH, A.N. \& CROCKETT, D.J. (1957). Nature of oedema in paralysed limbs of hemiplegic patients. British Medical Journal, 2, 1280.

HAWK, J.L.M. \& PARRISH, J.A. (1982). Responses of normal skin to ultraviolet radiation. In The Science of Photomedicine, Regan, J.D. \& Parrish, J.A. (Eds.), p. 219, Plenum Press: New York.
MULLEY, G. (1980). Axillary temperature differences in hemiplegia. Postgraduate Medical Journal, 56, 248.

STEINBROCKER, O. \& ARGYROS, T.G. (1958). Shoulderhand syndrome: present status as diagnostic and therapeutic entity. Medical Clinics of North America, 42, 1533.

WILlIAMS, D.I. (1984). Uneven tanning. British Medical Journal, 288, 243. 